\title{
Features of using human capital in agrarian sector of economy
}

\author{
G. P. Butko ${ }^{1,2 \bowtie}$, O. N. Saparova ${ }^{3}$ \\ ${ }^{1}$ Ural State Forestry University, Ekaterinburg, Russia \\ ${ }^{2}$ Ural State Agrarian University, Ekaterinburg, Russia \\ ${ }^{3}$ Ural State Mining University, Ekaterinburg, Russia \\ E-mail: gpbutko@mail.ru
}

\begin{abstract}
The article discusses the influence of human capital on the efficiency of the agro-industrial complex. The purpose of the study is to analyze the use and management of human capital from the standpoint of practice, the development of systems that are used to manage and develop employees of an organization. Results. The article reveals the results of the analysis of the development level of the agro-industrial complex and the state of the social infrastructure of the region as the most important conditions for the formation of human capital, functioning within a particular region. On the basis of theoretical generalizations, the analysis of statistical data, the desired dependencies and the rating of countries according to the human development index were obtained. The state and development of human capital is based on the initial data of Russian agricultural organizations for 2017-2019. As a result of the analysis, two types of models were proposed - linear and nonlinear models; correlation and regression analysis of statistical data was carried out. The main importance is the setting of the prospects for the development of human capital and, on this basis, the possibility of creating and strengthening food self-sufficiency. In the formation of human capital, the topical issue is the qualifications of specialists and its constant growth. The article presents in the most detail the results of processing the theory of statistical information on the example of correlation-regression analysis. Scientific novelty. The results obtained in the process of work have theoretical novelty and practical value, because, first of all, in the context of a pandemic, the formation of the importance of an emphasis on the progressiveness of the regulatory and legal framework and the improvement of stimulating the demand for highly qualified specialists in rural areas has been accomplished. Achieving competitive advantages is possible on the basis of sustainable development as a factor in ensuring economic stability. The structure of the process of using human capital includes progressive elements based on a balance between the increase and depletion of available resources. The results of the study confirmed the significance of the proposed indicators of human capital, however, they refuted the hypothesis of a direct relationship between the number of employees with higher education and the efficiency of the agro-industrial complex.
\end{abstract}

Keywords: human development index, methods for assessing human capital, agriculture, human capital, intellectual capital, correlation and regression analysis, performance indicators.

For citation: Butko G. P., Saparova O. N. Features of the use of human capital in the agricultural sector of the economy // Agrarian Bulletin of the Urals. 2021. No. 09 (212). Pp. 73-79. DOI: 10.32417/1997-4868-2021-212-09-73-79.

Date of paper submission: 02.08.2021, date of review: 06.08.2021, date of acceptance: 11.08.2021.

\section{Introduction}

The economic and political situation both at the state and international levels brings topical issues to the fore. These include improving the efficiency of the agro-industrial complex (AIC). The category of human capital is presented in the program documents of the Russian Federation. This is evidenced by the St. Petersburg International Economic Forum, where it was emphasized that the problem of human resource management has a special role.

As studies show, ... "sustainable and dynamic development of rural areas has become an object of federal state policy only in recent years, while previously practiced strategies most often focus on purely production aspects (issues of intensification of agricultural production, first of all). And, on the contrary, they exacerbate both ecological and social imbalances in society" [11, $p$. 67]. They are trying to study the influence of human and social capital on entrepreneurial activity [13]. Equally important are the issues of the evolutionary theory of human capital [17]. The author's hypothesis is that the situation with human resource management at agro-industrial complex enterprises has worsened in the context of a pandemic, and the need for a systematic approach to human resource management has intensified, highlighting their leading role in achieving an effective result in the innovative environment of the organization. 


\section{Methods}

Research methods: economic analysis, monographic analysis, system analysis.

A feature of the analysis of the use and management of human capital is the combination of such components as modern technologies with advanced methodology for the formation, management and development of human capital to achieve and maintain competitive advantages.

\section{Results}

Human capital management refers to HR services, recruiting, human capital assessment, service verification, talent management, human resources management, outsourcing, human capital management solutions software.

In addition, the "analysis of the use and management of human capital" refers to the practice, processes and systems that are used to manage and develop the employees of the organization ("assets") [1].

As a rule, in management, the human component is the most burdensome of all assets on the one hand, and on the other, people are the only element that has the ability to produce the value of a product. The performance of an organization's human capital is not always predictable and is under the control of the employer. Human capital management practices are based on the belief that labor is part of a company's capital, just like other assets such as land, buildings and equipment.

A special place in the formation of human capital is occupied by the qualifications of specialists. With the growing economy of the agricultural sector, many agricultural enterprises are acutely aware of the lack of labor with secondary and higher education. This is aggravated by low wages of employees and unsatisfactory working conditions in rural areas [2]. The importance of foreign approaches to assessing the human capital of the agroindustrial complex of enterprises should be confirmed. As the research has shown, there have been many attempts to estimate the value of human capital using these methods and various techniques. Assessment of the structure of labor motivation at enterprises of the agro-industrial complex and the direct transformation of the concept of "Labor" is considered in the author's works [8], [9], [11-18]. The cost of human capital is, in our opinion, not just the price of productive abilities, but above all an integrated value, adjusted for the number of these abilities. They were one of the first to calculate the value of human capital in the United States in 1961. Experts used the following method: the cost of one year of education at each level was multiplied by the number of person-years of education accumulated by the population at a given point in time.

N. I. Pirozhkova believes that thanks to the economic analysis of the human capital of an enterprise, it will be possible to understand from a scientific point of view managerial decisions and actions at the enterprise, the socio-economic policy at the enterprise, as well as economic analysis will contribute to the choice of the best options for action [10]. She allocated individual, collective and social human capital.

However, all the increasing sanctions from the Western world unequivocally make us understand that the country's food security depends on the effective use of human capital, among other things.

Most scientists consider the most important factor in the development of any economy is to be effective management of human capital. The transition of the economy to innovative development has revealed the importance of human capital development in agriculture in Russia, the use of management techniques that can increase production efficiency. The leading parameter of human capital is the health of people determined by various natural and infrastructural circumstances, which determines their ability to work fully. In this regard, for the specification of some parameters of human capital, the above techniques are used [11], [12],

Russia is not included in the group of countries with a very high HDI level, and in the group of countries with a high level of HDI development it occupies not the most honorable $52^{\text {nd }}$ place, between Kazakhstan $\left(51^{\text {st }}\right.$ place $)$ and Belarus (53 ${ }^{\text {rd }}$ place) [3].

Human Development Index 2019

\begin{tabular}{|c|c|c|c|c|c|}
\hline Rating & $H D I$ & Country & Rating & $H D I$ & Country \\
\hline \multicolumn{3}{|c|}{ Countries with very high HDI levels (over 0.9) } & \multicolumn{3}{|c|}{ Countries with an average HDI level (from 0.5 to 0.8 ) } \\
\hline 1 & Norway & 0.957 & 69 & Albania & 0.795 \\
\hline 2 & Switzerland & 0.955 & 70 & Cuba & 0.783 \\
\hline 6 & Germany & 0.947 & 74 & Ukraine & 0.779 \\
\hline 22 & Slovenia & 0.917 & 142 & Nepal & 0.602 \\
\hline 26 & France & 0.901 & 166 & Tanzania & 0.529 \\
\hline \multicolumn{3}{|c|}{ Countries with high HDI levels (0.8 to 0.9) } & \multicolumn{3}{|c|}{ Countries with a low HDI level (less than 0.5) } \\
\hline 29 & Estonia & 0.892 & 173 & Ethiopia & 0.485 \\
\hline 43 & Chile & 0.851 & 181 & Mozambique & 0.456 \\
\hline 51 & Kazakhstan & 0.825 & 184 & Mali & 0.434 \\
\hline 52 & Russia & 0.824 & 187 & Chad & 0.397 \\
\hline 53 & Belarus & 0.823 & 189 & Niger & 0.394 \\
\hline
\end{tabular}

Compiled by the authors based on the United Nations Development Program: Human Development Index 2020. 


\section{Agrarian Bulletin of the Urals No. 09 (212), 2021}

It is important to emphasize that an insignificant part factors described above lead to a decrease in the natural of Russia's human capital is concentrated in agriculture, base for the formation of human capital in the agricultural and this share is constantly decreasing. Thus, over 13 years of research, the proportion of the population employed in agriculture and forestry, hunting and fishing decreased from $19.70 \%$ in 2005 to $11.7 \%$ in 2018 (by $8 \%$ ). This is due to low wages, job cuts and, as a result, unemployment. An important role is played by the migration of rural residents of working age to cities, and this is a tendency for many decades. However, it is necessary to note some stabilization of the process of change in the size of the rural population, since 2014 the percentage of people employed in agriculture has fluctuated around $12 \%$ of the total number of people employed, the decrease occurs by no more than $1 \%$ per year [6].

The decline in the number of employed people in agriculture is a natural process, which is conditioned by the transition to an innovative way of development. All the

sector of Russia [4], [5], [6], [14].

At the same time, it should be noted that agriculture has large unused reserves and is one of the priority sectors of the country's economy, since it plays a significant role in preserving rural society, in solving employment problems, generating income, preserving and increasing human capital. The agro-industrial complex is also of great importance in ensuring the country's food security.

In 2018, only 4267 thousand people worked in the agricultural sector, which is $11.7 \%$ of the total employed population. The nominal gross wages of agricultural workers are constantly growing (2010 - 11109 rubles, $2018-28699$ rubles or as a percentage of the previous year $2010-110.0 \%, 2018-111.8 \%$ ), real wages also increased, but somewhat less [4], [5], [15].

Table 2

Dynamics of the share and number of population employed in agriculture, forestry, hunting and fishing in 2012-2018

\begin{tabular}{|c|c|c|c|c|c|c|c|c|c|c|c|c|c|c|c|c|}
\hline & \multicolumn{2}{|c|}{2005} & \multicolumn{2}{|c|}{2010} & \multicolumn{2}{|c|}{2014} & \multicolumn{2}{|c|}{2015} & \multicolumn{2}{|c|}{2016} & \multicolumn{2}{|c|}{2017} & \multicolumn{2}{|c|}{2018} & \multicolumn{2}{|c|}{2019} \\
\hline \multicolumn{17}{|c|}{ Russia } \\
\hline & $m$ & $f$ & $m$ & $f$ & $m$ & $f$ & $m$ & $f$ & $m$ & $f$ & $m$ & $f$ & $m$ & $f$ & $m$ & $f$ \\
\hline $\begin{array}{l}\text { \% to } \\
\text { employed }\end{array}$ & 11.9 & 7.8 & 9.6 & 5.5 & 8.0 & 5.1 & 7.9 & 5.1 & 8,0 & 5,0 & 7,7 & 4,0 & 7,7 & 4,0 & 7,6 & 4,0 \\
\hline $\begin{array}{l}\text { Employed, } \\
\text { thousand } \\
\text { people }\end{array}$ & 4115 & 2643 & 3404 & 1898 & 2911 & 1787 & 2941 & 1784 & 2985 & 1752 & 2850 & 1418 & 2858 & 1409 & 2793 & 1403 \\
\hline \multicolumn{17}{|c|}{ Germany } \\
\hline $\begin{array}{l}\text { \% to } \\
\text { employed }\end{array}$ & \multicolumn{2}{|c|}{1.6} & \multicolumn{2}{|c|}{1.5} & \multicolumn{2}{|c|}{1.5} & \multicolumn{2}{|c|}{1.6} & \multicolumn{2}{|c|}{1.6} & \multicolumn{2}{|c|}{1.4} & \multicolumn{2}{|c|}{1.3} & \multicolumn{2}{|c|}{1.4} \\
\hline $\begin{array}{l}\text { Employed, } \\
\text { thousand } \\
\text { people }\end{array}$ & \multicolumn{2}{|c|}{642.0} & \multicolumn{2}{|c|}{630.3} & 63 & 4.9 & \multicolumn{2}{|c|}{640.6} & \multicolumn{2}{|c|}{661.9} & \multicolumn{2}{|c|}{662.3} & \multicolumn{2}{|c|}{663} & \multicolumn{2}{|c|}{664} \\
\hline \multicolumn{17}{|c|}{ Kazakhstan } \\
\hline $\begin{array}{l}\text { \% to } \\
\text { employed }\end{array}$ & \multicolumn{2}{|c|}{19.2} & \multicolumn{2}{|c|}{19.0} & \multicolumn{2}{|c|}{18.9} & \multicolumn{2}{|c|}{18.0} & \multicolumn{2}{|c|}{16.2} & \multicolumn{2}{|c|}{16} & \multicolumn{2}{|c|}{14.1} & \multicolumn{2}{|c|}{14.5} \\
\hline $\begin{array}{l}\text { Employed, } \\
\text { thousand } \\
\text { people }\end{array}$ & 21 & 50 & 21 & 96 & 160 & 5.1 & 15 & 3.4 & 138 & 5.5 & & 00 & & 00 & & 15 \\
\hline & & & & & & & Ukra & ine & & & & & & & & \\
\hline $\begin{array}{l}\text { \% to } \\
\text { employed }\end{array}$ & & .9 & 18 & .7 & 17 & .5 & & .1 & 17 & .1 & & 7.5 & & 8 & & 7.9 \\
\hline $\begin{array}{l}\text { Employed, } \\
\text { thousand } \\
\text { people }\end{array}$ & 41 & 56 & 39 & 98 & 33 & 89 & $30 s$ & 1.4 & 307 & 0.3 & & 20 & & 00 & & 31 \\
\hline
\end{tabular}

Table 3

Change in average monthly wages (as a percentage of the previous year)

\begin{tabular}{|c|c|c|c|c|c|c|}
\hline & 2010 & 2015 & 2016 & 2017 & 2018 & 2019 \\
\hline \multicolumn{7}{|c|}{ Nominal accrued wages } \\
\hline Total across Russia & 112.4 & 105.1 & 107.9 & 106.7 & 111.6 & 106.7 \\
\hline Agriculture, forestry, hunting, fishing and fish farming & 110.0 & 112.6 & 110.9 & 112.0 & 111.8 & 112.0 \\
\hline \multicolumn{7}{|c|}{ Real accrued wages } \\
\hline Total across Russia & 105.2 & 91.0 & 100.8 & 102.9 & 108.5 & 108.5 \\
\hline Agriculture, forestry, hunting, fishing and fish farming & 103.0 & 97.4 & 103.6 & 108.1 & 108.7 & 108.7 \\
\hline \multicolumn{7}{|c|}{$\begin{array}{c}\text { Average monthly nominal accrued wages of employees of organizations } \\
\text { by economic activity (rubles) }\end{array}$} \\
\hline Total across Russia & 20952 & 34030 & 36709 & 39167 & 43724 & 47468 \\
\hline Agriculture, forestry, hunting, fishing and fish farming & 11109 & 20670 & 22915 & 25671 & 28699 & 31581 \\
\hline
\end{tabular}


Аграрный вестник Урала № 09 (212), 2021 г.

Table 4

The number of employed by education level and types of economic activity in 2019 [7]

\begin{tabular}{|c|c|c|c|c|c|c|c|}
\hline & \multirow{3}{*}{$\begin{array}{c}\text { Total, } \\
\text { thousand } \\
\text { people }\end{array}$} & \multicolumn{6}{|c|}{ Including have education } \\
\hline & & \multirow[t]{2}{*}{ Higher } & \multicolumn{2}{|c|}{ Secondary vocational } & \multirow{2}{*}{$\begin{array}{l}\text { Average } \\
\text { general }\end{array}$} & \multirow{2}{*}{$\begin{array}{c}\text { Main } \\
\text { common }\end{array}$} & \multirow{2}{*}{$\begin{array}{c}\text { Have } \\
\text { no basic } \\
\text { common }\end{array}$} \\
\hline & & & $\begin{array}{l}\text { On the } \\
\text { training } \\
\text { program for } \\
\text { mid-level } \\
\text { specialists }\end{array}$ & $\begin{array}{c}\text { According to } \\
\text { the qualification } \\
\text { training program. } \\
\text { workers, } \\
\text { employees }\end{array}$ & & & \\
\hline \multicolumn{8}{|c|}{ In thousands of people } \\
\hline $\begin{array}{l}\text { Employed - total, } \\
\text { including }\end{array}$ & 71933 & 24632 & 18423 & 13951 & 12121 & 2635 & 172 \\
\hline $\begin{array}{l}\text { Agriculture, forestry, } \\
\text { hunting, fishing and } \\
\text { fish farming }\end{array}$ & 4196 & 541 & 784 & 973 & 1310 & 527 & 61 \\
\hline \multicolumn{8}{|c|}{ In percents } \\
\hline $\begin{array}{l}\text { Employed - total, } \\
\text { including }\end{array}$ & 100 & 34.2 & 25.6 & 19.4 & 16.9 & 3.7 & 0.2 \\
\hline $\begin{array}{l}\text { Agriculture, forestry, } \\
\text { hunting, fishing and } \\
\text { fish farming }\end{array}$ & 100 & 12.9 & 18.7 & 23.2 & 31.2 & 12.5 & 1.5 \\
\hline
\end{tabular}

Table 5

Growth (decline) rates of labor productivity by type of economic activity (as a percentage of the previous year) [7]

\begin{tabular}{|l|c|c|c|c|}
\hline & $\mathbf{2 0 1 5}$ & $\mathbf{2 0 1 6}$ & $\mathbf{2 0 1 7}$ & $\mathbf{2 0 1 8}$ \\
\hline Total & 98.7 & 100.1 & 102.1 & 102.8 \\
\hline Agriculture, forestry, hunting, fishing and fish farming & 104.0 & 102.2 & 105.3 & 102.8 \\
\hline
\end{tabular}

Table 6

Turnover of organizationsby type of economic activity (thousand rubles) [7]

\begin{tabular}{|l|c|c|c|c|}
\hline & $\mathbf{2 0 1 6}$ & $\mathbf{2 0 1 7}$ & $\mathbf{2 0 1 8}$ & 2019 \\
\hline Total & 146376.8 & 158778.0 & 191813.3 & 201315.5 \\
\hline Agriculture, forestry, hunting, fishing and fish farming & 2640.5 & 2720.4 & 3160.7 & 3418.0 \\
\hline \multicolumn{7}{|c|}{ Agriculture, forestry, hunting, fishing and fish farming } \\
\hline Total & 4520.3 & 4561.7 & 4214.7 & 3826.9 \\
\hline Agriculture, forestry, hunting, fishing and fish farming & 130.0 & 129.4 & 115.4 & 102.9 \\
\hline
\end{tabular}

These indicators are quite comparable with the aver- economy. This is especially evident if we study the probage indicators for Russia, which include changes in nomi- lems of the productive use of human capital, the methnal and real wages in all sectors.

According to the latest data (May 2021), the number of vacancies in the group of occupations "agriculture" has increased. The largest increase in demand is observed in the Novosibirsk region: from March 2019 to March $2020-200 \%$, followed by the Sverdlovsk region $183 \%$. Throughout Russia, the activity of employers in this group of professions increased by $115 \%$. Most in demand are agronomists, machine operators, tractor drivers, greenhouse workers, gardeners, vegetable growers and agricultural engineers. The average salary offered by employers in agriculture in 2020 is 35000 rubles per month [6].

In modern conditions, the role of the educational and qualification component of human resources in the agricultural sector is increasing - the creation of such workers who have the necessary knowledge and skills in the technical, agronomic, and economic spheres [6].

It should be noted that the current level of human capital development in agriculture is insufficient to fully meet the needs of Russia and integration into the world odological foundations of the study of the relationship between the quality of personnel and the effectiveness of its use [9], [10]. Hence the need for a more detailed study.

Human capital, being an independent part of the population, from the standpoint of economic and production approaches, largely depends on the socio-cultural factors of the formation environment [18].

In accordance with the author's assumptions and analytical assessment, the following factors affect the efficiency of using human capital:

1. Professional level of employees (education level).

2. Wages of employees.

3. Labor productivity of workers.

In order to confirm or deny this hypothesis, a correlation and regression analysis of the initial data is made.

I. First of all, we will consider the data of correlation analysis. The correlation coefficient is a quantitative characteristic of the degree of relationship between two properties of objects. The calculations were carried out according to the formula (1) below and are presented below (Table 7). 
The value of the coefficient r (correlation)

\begin{tabular}{|l|c|c|c||}
\hline \multicolumn{1}{|c|}{} & $\begin{array}{c}\text { Average monthly } \\
\text { nominal salary, rubles }\end{array}$ & $\begin{array}{c}\text { Nominal accrued } \\
\text { wages fee, \% }\end{array}$ & $\begin{array}{c}\text { Real accrued wages, } \\
\text { \% }\end{array}$ \\
\hline 1 & 2 & 3 & 4 \\
\hline $\begin{array}{l}\text { Hecondary vocational training } \\
\text { program for mid-level specialists }\end{array}$ & 0.32364 & & \\
\hline $\begin{array}{l}\text { Secondary vocational training } \\
\text { program qual. workers, employees }\end{array}$ & 0.99504 & & \\
\hline Average general & 0.94629 & & \\
\hline Main common & 0.99583 & & \\
\hline Have no basic common & 0.87944 & & \\
\hline Share of employed in agriculture, $\%$ & 0.63005 & & \\
\hline $\begin{array}{l}\text { Change in labor productivity,\% to the } \\
\text { previous year }\end{array}$ & & 0.04046 & \\
\hline
\end{tabular}

$$
r=\frac{\sum_{i=1}^{n}\left(x_{i}-\bar{x}\right) \cdot\left(y_{i}-\bar{y}\right)}{\sqrt{\sum_{i=1}^{n}\left(x_{i}-\bar{x}\right)^{2}} \cdot \sqrt{\sum_{i=1}^{n}\left(y_{i}-\bar{y}\right)^{2}}} .
$$

As follows from the analysis, according to the data of the average monthly nominal wages in the table below it can be seen that the wages offered in agriculture are attractive for people with less than higher education, and those with higher education do not consider this job attractive, at least measure according to wages. It follows from the third column that the number of people employed in agriculture does not depend on wages, and the fourth says that labor productivity does not depend very much on the level of wages.

II. The regression model included factors similar to the previous ones, taken from the tables above:

$y$ - effective indicator; the average annual income of enterprises from the sale of products.

$x_{1}$ - factorial sign; average annual salary of one employee;

$x_{2}$ - factorial feature; the proportion of highly qualified (with higher education) workers in the total number of agricultural workers;

$x_{3}$ - factorial feature; the proportion of skilled workers (with secondary vocational education under the program for training mid-level specialists and secondary vocational education under the program for the training of skilled workers, office workers) in the total number of agricultural workers.

As a result of the regression analysis, the following linear regression equation was obtained:

$$
y=a_{0}+a_{1} x_{1}+a_{2} x_{2}+a_{3} x_{3}
$$

Based on the performed regression analysis, the following data were obtained:

II.1. Analysis of the dependence of "the average annual income of enterprises from the sale of products" "the average annual salary of one employee".

$\mathrm{R}$-square is the coefficient of determination in our example -0.952 , or $95.2 \%$. This means that the calculated parameters of the model explain the relationship between the income of agricultural enterprises and average wages by $95.2 \%$.
II.2. Analysis of the dependence of "the average annual income of enterprises from the sale of products" - "the proportion of highly qualified (with higher education) workers in the total number of agricultural workers".

In the second group, according to the results of regression analysis, we can say that there is no relationship between the income of an agricultural enterprise and the number of employees with higher education, that is, the component of human capital that is characterized by the presence of higher education in agriculture is not significant.

II.3. Analysis of the dependence of "the average annual income of enterprises on the sale of products" - "the proportion of qualified workers (with secondary vocational education under the program for training mid-level specialists and secondary vocational education under the program for the training of skilled workers, office workers) in the total number of agricultural workers".

However, the relationship between the income of agricultural enterprises and employees with secondary education is significant, which can be seen on the basis of the following data (Table 10).

In this case, the R-squared parameter is 0.890 or $89.0 \%$, this high value of the coefficient of determination tells us about the close dependence of secondary specialized education and the income of agricultural enterprises.

It should be noted that when shaping the personnel policy at agricultural enterprises, it is necessary to take into account the need not only for material incentives for labor, but also other factors that can affect the motivation of workers, especially those with higher education (and, accordingly, a higher human development index). At the same time, it is necessary to take into account the fact that workers with secondary vocational education have the greatest influence on the income of agricultural enterprises.

\section{Discussion and Conclusion}

As a result of the theoretical review and analysis, the main directions of the development and transformation of human capital in the agro-industrial complex were determined. It is confirmed that the lack of human capital in general, as well as the demanded quality in particular, is aggravated by the ongoing migration of the population from rural areas to cities and towns. 
Аграрный вестник Урала № 09 (212), 2021 г.

Table 8

Regression statistics of the $1^{\text {st }}$ stage of the analysis

\begin{tabular}{|c|c|}
\hline \multicolumn{2}{|c|}{ Regression statistics for $x_{1}$} \\
\hline Multiple R & 0.975747775 \\
\hline$R$-square & 0.952083721 \\
\hline Normalized R-square & 0.928125582 \\
\hline Standard error & 98.75337565 \\
\hline Observations & 4 \\
\hline
\end{tabular}

Table 9

Regression statistics of the $2^{\text {nd }}$ stage of analysis

\begin{tabular}{|l|c|}
\hline \multicolumn{2}{|c|}{ Regression statistics for $\boldsymbol{x}_{2}$} \\
\hline Multiple $R$ & 0.270833615 \\
\hline$R$-square & 0.073350847 \\
\hline Normalized R-square & -0.389973729 \\
\hline Standard error & 434.2782449 \\
\hline Observations & 4 \\
\hline
\end{tabular}

Table 10

Regression statistics of the $3^{\text {rd }}$ stage of analysis

\begin{tabular}{|l|c|}
\hline \multicolumn{2}{|c|}{ Regression statistics for $\boldsymbol{x}_{\mathbf{3}}$} \\
\hline Multiple $R$ & 0.943383929 \\
\hline -square & 0.889973237 \\
\hline Normalized R-square & 0.834959856 \\
\hline Standard error & 149.6440919 \\
\hline Observations & 4 \\
\hline
\end{tabular}

A feature of the rural areas of the Sverdlovsk region, ment in that personnel management is understood as relike other regions of the Russian Federation, is a nega- cruiting personnel, calculating wages, benefits, as well as tive factor in the provision and possibility of obtaining other fundamental aspects of labor relations. Based on the comfortable housing and its insufficiently high quality in analysis of the concepts presented and the calculations comparison with modern urban living standards.

A feature of the analysis of the use and management criterion of human capital is the income that it (human of human capital is that it fundamentally differs from the capital) brings to a person, the organization of the agrotraditional concept of analysis and personnel manage- industrial complex as a whole.

\section{References}

1. Gorbunova O. S. Formirovanie chelovecheskogo kapitala agrarnoy sfery regiona: avtoreferat dis. ... kand. ekon. nauk [Formation of human capital of the agrarian sphere of the region: abstract dis. ... candidate economic sciences]. Ekaterinburg, 2018. 28 p. (In Russian.)

2. Petrov L. M. Upravlenie chelovecheskim kapitalom na predpriyatiyakh agropromyshlennogo kompleksa v usloviyakh global'nykh izmeneniy [Human capital management at enterprises of the agro-industrial complex in the context of global changes] // Creative Economy. 2021. Vol. 15. No. 2. Pp. 443-458. DOI: 10.18334/ce.15.2.111574. (In Russian.)

3. Reyting stran mira po indeksu chelovecheskogo razvitiya [Rating of countries in the world according to the human development index] [e-resource]. URL: https://gtmarket.ru/ratings/human-development-index (date of reference: 15.04.2021). (In Russian.)

4. Trud i zanyatost' v Rossii. 2019: statatisticheskiy sbornik [Labor and employment in Russia. 2019: Statistical digest]. Moscow: Rosstat, 2019. 135 p. (In Russian.)

5. Trud i zanyatost' v Rossii. 2017: statatisticheskiy sbornik [Labor and employment in Russia. 2017: Statistical digest]. Moscow: Rosstat, 2017. 261 p. (In Russian.)

6. Rabochaya sila, zanyatost' i bezrabotitsa v Rossii (po rezul'tatam vyborochnykh obsledovaniy rabochey sily). 2020: statatisticheskiy sbornik [Labor force, employment and unemployment in Russia (based on the results of sample surveys of the labor force). 2020: Statistical digest]. Moscow: Rosstat, 2020.145 p. (In Russian.) 


\section{Agrarian Bulletin of the Urals No. 09 (212), 2021}

7. Rossiya v tsifrakh. 2020: Kratkiy statatisticheskiy sbornik [Russia in numbers. 2020: Statistical digest]. Moscow: Rosstat, 2020.550p. (In Russian.)

8. Butko G. P., Saparova O. N. Transformatsiya ponyatiya "Trud" [Transformation of the concept of "Labor"] // Teoriya i praktika mirovoy nauki. 2020. No 12. Pp. 2-6. (In Russian.)

9. Butko G. P., Saparova O. N. Otsenka struktury motivatsii truda na predpriyatiyakh agropromyshlennogo kompleksa [Assessment of the structure of labor motivation at the enterprises of the agro-industrial complex] // Ekonomika sel'skogo khozyaystva Rossii. 2020. No. 8. Pp. 73-79. DOI: 10.32651/208-73. (In Russian.)

10. Pirozhkova N. I. Klassifikatsiya chelovecheskogo kapitala predpriyatiya // Ekonomika: vchera, segodnya, zavtra [Classification of human capital of an enterprise] // Economics: Yesterday, Today and Tomorrow. 2011. No. 2. Pp. 136-145. (In Russian.)

11. Golovina S. G., Mikolaychik I. N., Smirnova L. N. Sotsial'no-ekonomicheskiye usloviya razvitiya chelovecheskogo kapitala v sel'skoy mestnosti [Socio-economic conditions for the development of human capital in rural areas] // Agrarian Bulletin of the Urals. 2020. No. 8. Pp. 65-79. DOI: 10.32417/1997-4868-2020-199-8-65-79. (In Russian.) 12. Wolz A., Golovina S., Nilsson J., Hess S. Reviewing Changing Institutional Conditions for Private Farming in Russia // Outlook on Agriculture. 2016. № 45 (2). P. 111-116. DOI: 10.1177/0030727016651214.

13. Chitsaz E., Tajpour M., Hosseini E., Khorram H., Zorrieh S. The Effect of Human and Social Capital on Entrepreneurial Activities: A Case Study of Iran and Implications // Entrepreneurship and Sustainability. 2019. Iss. 6 (3), Pp. 1193-1203. DOI: 10.9770/jesi.2019.6.3(24).

14. Bombiak E. Green human resource management - the latest trend or strategic necessity? // Entrepreneurship and Sustainability. 2019. Iss. 6 (4). Pp. 1647-1662. DOI: 10.9770/jesi.2019.6.4(7).

15. Golovina S., Hess S., Nilsson J., Wolz A. Networking among Russian farmers and their prospects for success. Post-Communist Economies. 2019. Vol. 31. No. 4. Pp. 484-499. DOI: 10.1080/14631377.2018.1537737.

16. Zhou B., Liu W., Lu W., Zhao M., Li L. Application of OECD LSE Framework to Assess Spatial Differences in Rural Green Development in the Arid Shaanxi Province, China // International Journal of Environmental Research and Public Health. 2019. No. 17 (1). P. 286. DOI: 10.3390/ijerph17010286.

17. Canibano C., Potts J. Toward an evolutionary theory of human capital // Journal of Evolutionary Economics. 2019. Vol. 29 (3). Pp. 1017-1035. DOI: 10.1007/s00191-018-0588-y.

18. Voronin B. A., Chupina I. P., Voronina Ya. V., Fateyeva N. B. Kachestvo zhizni i chelovecheskiy kapital v sel'skoy mestnosti Rossii [Quality of life and human capital in rural areas of Russia] // Ekonomika sel'skogo khozyaystva. 2020. No. 8. Pp. 96-102. DOI: 10.32651/208-96. (In Russian.)

\section{Authors'information:}

Galina P. Butko ${ }^{1,2}$, doctor of economic sciences, professor, ORCID 0000-0003-0304-1265, AuthorID 657031;

+79022591135,gpbutko@mail.ru

Olga N. Saparova ${ }^{3}$, senior lecturer, ORCID 0000-0002-5565-2298, AuthorID 251300; +7912 2623607 , on2002@bk.ru

${ }^{1}$ Ural State Forestry University, Ekaterinburg, Russia

${ }^{2}$ Ural State Agrarian University, Ekaterinburg, Russia

${ }^{3}$ Ural State Mining University, Ekaterinburg, Russia 
Участие местных сообществ

в развитии сельских территорий: опыт Европейского союза

\author{
С. Г. Головина ${ }^{1,3 凶}$, А. В. Ручкин ${ }^{1}$ И. Н. Миколайчик ${ }^{2}$, Л. Н. Смирнова ${ }^{2}$ \\ ${ }^{1}$ Уральский государственный аграрный университет, Екатеринбург, Россия \\ ${ }^{2}$ Курганская государственная сельскохозяйственная академия имени Т. С. Мальцева, \\ Лесниково, Россия \\ ${ }^{3}$ Российский государственный аграрный заочный университет, Москва, Россия \\ ¿E-mail: s_golovina@yahoo.com
}

Аннотация. Использование опыта реализации Единой сельскохозяйственной политики (САР) в государствах - членах Европейского союза (EC) актуально как для России, так и для других стран мира, заинтересованных в успешном развитии аграрной отрасли экономики и сельских территорий, роль которых в достижении национальной безопасности стран и регионов (продовольственной, биологической, экологической и прочей) существенно возрастает в связи с возникшими сегодня вызовами и угрозами (изменение климата, пандемия COVID-19, обострение международной обстановки). Цель исследования, результаты которого представлены в данной статье, направлена на тщательное изучение особого подхода, реализуемого в рамках CAP, именуемого в европейском законодательстве LEADER/CLLD и означающего тесное сочетание всестороннего межотраслевого взаимодействия с активным участием местных сообществ в сельском развитии. В работе применены обзорно-аналитические методы исследования, с помощью которых скрупулезному изучению подлежали: 1) действующее (имеющее отношение к теме исследования) законодательство, 2) реализуемые в странах членах ЕС программы, 3) значимые научные публикации. Результатом работы стали обзорноаналитические выводы и практические рекомендации относительно перспектив использования различных аспектов LEADER/CLLD в отечественной политической и хозяйственной практике, учитывая, что опыт местных жителей в сочетании с мнением других заинтересованных сторон способен помочь лучше адаптировать политику развития сельских территорий к реальным потребностям и возможностям, а в границах сельских сообществ сформировать специфический (уникальный) человеческий капитал, включающий, помимо особых профессиональных навыков, 1) способность выступать с конструктивными инициативами; 2) обладание чувством местной идентичности и ответственности за свою деятельность; 3) умение участвовать на равных с другими партнерами в определении стратегий местного развития; 4) доверие между людьми, частными предприятиями, государственными учреждениями и отраслевыми сообществами, заинтересованными в успешном сельском развитии, формирующееся в процессе постоянного взаимодействия. Научной новизной обладают теоретические и практические выводы, касающиеся содержания инициатив LEADER/CLLD, а также заключения, связанные с возможностями внедрения инструментов и механизмов поддержки сельских территорий, реализуемых непосредственно с привлечением местных сообществ при финансовой поддержке государства. Ключевые слова: Единая сельскохозяйственная политика, Европейский союз, сельские территории, LEADER/ CLLD, вызовы и угрозы.

Для цитирования: Головина С. Г., Ручкин А. В., Миколайчик И. Н., Смирнова Л. Н. Участие местных сообществ в развитии сельских территорий: опыт Европейского союза // Аграрный вестник Урала. 2021. № 09 (212). C. 80-92. DOI: 10.32417/1997-4868-2021-212-09-80-92.

Дата поступления статьи: 26.07.2021, дата рецензирования: 03.08.2021, дата принятия: 09.08.2021.

Постановка проблемы (Introduction)

Единая сельскохозяйственная политика (САР), реализуемая в странах - членах Европейского союза (ЕС) с 1962 г., отличается тем, что предусмотренные в ее рамках мероприятия реализуются с опорой, вопервых, на фермеров и других участников аграрного производства, выполняющих разнообразные эконо- мические, социальные, экологические функции в границах сельского пространства, во-вторых, на местные сообщества, играющие исторически значимую роль в сельской экономике и сельском развитии. Не случайно в реализации второго компонента СAP (Second Pillar), финансирование которого осуществляется через Европейский фонд развития сельских терри- 


\section{Agrarian Bulletin of the Urals No. 09 (212), 2021}

торий (EAFRD), меры, связанные непосредственно с программами LEADER (Liaison Entre Actions De Développement De L'économie Rurale) и CLLD (Community-Led Local Development), занимают в общей совокупности мер САР самый большой удельный вес. Необходимо отметить, что если сама программа LEADER реализуется в EC на протяжении 30 лет, то потребность и стремление к сотрудничеству и кооперации, особенно в сельском пространстве, формируется в европейских странах веками.

Сегодня такая (основанная на местном участии) концепция решения проблем села (сельских территорий) аргументируется не только практическими достижениями, но и результатами исследований современной науки, в частности такими ее теоретическими подходами, как:

1) пространственный подход к развитию регионов (Area-Based Development Approach - ABD);

2) комплексное (интегрированное) развитие сельских районов (Integrated Rural Development - IRD);

3) территориальное развитие на основе консенсуса интересов участников (Participatory Negotiated Territorial Development - PNTD);

4) развитие, управляемое сообществами (CDD Community Driven Development) [1].

Каждый из выделенных подходов по-своему уникален и целесообразен, поэтому, синтезируя их основные идеи, можно выделить несколько особо важных аспектов. К примеру, ABD-подход ценен для развития сельских территорий тем, что предназначен для исследования конкретных географических районов (имеющих специфические проблемы в развитии) на основе комплексности, универсальности, участия, гибкости, принимая в расчет то, что каждый регион, с одной стороны, встроен в более широкое территориальное пространство, а с другой - имеет собственные задачи в области развития. В результате рассматриваемый $\mathrm{ABD}$-подход, концентрируясь на целых сообществах (а не на их сегментах или отдельных лицах), учитывает интересы не только отдельных категорий потенциальных бенефициаров, а всего населения, проживающего в границах той или иной территории. Относительно сельского развития важно отметить, что современные программные пакеты устойчивого развития сельских территорий интегрируют проблемы охраны окружающей среды, экономические, социально-политические и институциональные вопросы, решение которых, по мнению представителей данного подхода, эффективнее в том случае, если оно инициируется локальной властью, а не центральной администрацией и, более того, адаптировано к культурному, социально-экономическому и политическому контексту [2]. В итоге генерировать максимальный уровень развития можно путем социальной мобилизации и использования внутреннего потенциала территорий через различные социальные программы, подобные программе (а точнее, целой концепции) LEADER.
Следующая теория - IRD (теория комплексного развития сельских районов), которая, по сути, является отправной точкой в достижении конвергенции развития отдельных территорий внутри региона и преодолении в границах каждой из них как экономических, так и социальных трудностей, причем комплексное развитие сельских территорий (согласно практическому опыту) из-за ограниченности ресурсов не может интерпретироваться как одновременное решение совокупности всех имеющихся проблем. В связи с этим в первую очередь решаются задачи по расширению потенциала и росту эффективности его использования, а затем - по улучшению социальных условий и развитию инфраструктуры села, созданию устойчивых и многосторонних связей между сельскими районами и городскими центрами. Безусловно, трудности неизбежны, но, как показывает опыт европейских стран, они преодолеваются опять же путем мобилизации инициатив на региональном уровне.

Данная тактика объясняется тем, что:

1) только на уровне региональной системы можно выработать реальные программы, основанные на существующем ресурсном потенциале и взаимосвязи его элементов;

2) технический и административный потенциал для реализации региональных программ расположен, как правило, на региональном уровне и лучше всего может быть мобилизован именно там;

3) интегрированные в рамках географического (административного) района компоненты его ресурсного потенциала приводят к экономии на масштабе и сокращению издержек реализации проектов регионального развития;

4) мобилизация и активное участие различных групп населения наилучшим образом могут быть организованы на уровне области (региона);

5) территориальный уровень обеспечивает лучшие возможности для интеграции существующего аграрного производства с современными технологиями и несельскохозяйственной деятельностью, а также для создания новых форм социальной организации производства и других видов деятельности.

В итоге в концепции комплексного развития сельских территорий местные инициативы занимают важное место, а ключевым аспектом планирования и реализации региональных программ является привлечение к этому процессу сельского населения, которое в других подходах позиционируется лишь в качестве объекта тех или иных проектов.

Целесообразно обозначить и такой разработанный ФАО (Food and Agriculture Organization) подход к региональному развитию (сельскому прежде всего), как территориальное развитие на основе консенсуса интересов (PNTD - Participatory Negotiated Territorial Development), который (как и отмеченные выше теории) предполагает включение в процесс развития местных участников (снизу вверх), изучение и учет их мнения, что является важным условием согласия в принятии решений, достигаемого не на базе сугу- 
|| бо технических или экономических аргументов, а на основе широких социально-политических представлений. Практическая значимость этого подхода заключается, прежде всего, в том, что, концентрируясь на мотивации заинтересованных сторон и, как следствие, на мобилизации местных ресурсов для территориального развития путем их децентрализации его концептуальные положения в итоге стимулируют (что немаловажно) конструктивный диалог и генерируют положительные социально-экономические изменения.

Не менее популярно в научном сообществе еще одно перспективное теоретическое направление - развитие, управляемое сообществами (CDD Community Driven Development), которое Всемирным банком определяется как подход, наделяющий местные сообщества широким контролем над планированием размещения инвестиционных ресурсов в целях местного (территориального) развития [3].

B основе CDD-подхода лежит предположение о том, что местное население (сообщества) лучше знает ответы на вопросы относительно того:

1) как можно улучшить их жизнь и увеличить средства для жизнеобеспечения;

2) каким образом эффективно использовать территориальные ресурсы для удовлетворения насущных потребностей [4].

Как итог, фокус именно CDD-подхода направлен на целевых бенефициаров, которые должны способствовать выявлению местных приоритетов и фактической реализации инициатив в области локального развития, причем либо непосредственно (поставляя ресурсы), либо опосредованно (участвуя в управлении и контроле), однако важно иметь в виду, что, в сущности, уровень местного участия может варьироваться от простого обмена информацией до активной реализации экономических, социальных, политических прав и возможностей различными группами сообществ. Таким образом, если применение данного подхода способствует общественному контролю над ресурсами территорий, то основная задача регионов заключается в том, чтобы включить в процессы их развития как можно больше групп населения, учитывая при этом, что каждая из их них оказывает разное влияние на процессы принятия решений, в связи с чем необходимо убедиться, что мнение даже «слабых» заинтересованных сторон принимается во внимание.

В заключение вступительной части отметим, что изложенные теоретические подходы к региональному развитию в европейских странах нашли свое практическое применение в особой концепции LEADER, реализуемой на протяжении последних 30 лет в странах - членах ЕС. Описание сути данного подхода к развитию сельских территорий, изложение особенностей его применения в разные периоды реализации Единой сельскохозяйственной политики ЕC, анализ эффективности применяемых в рамках LEADER мер - основные задачи исследования, результаты которого представлены в данной статье.

\section{Методология и методы исследования (Methods)}

С опорой на теоретические конструкции описанных выше научных концепций локального развития и имеющихся сегодня других актуальных подходов к развитию сельских территорий [5] ретроспективному анализу подлежали этапы развития программ ЕС, реализуемых в рамках Единой сельскохозяйственной политики и основанных на локальных инициативах и местных участниках.

Поддержка развития сельских территорий под руководством местных сообществ (LEADER) определена в качестве значимых мер соответствующими регламентами Европейского Парламента и Совета ЕС, в связи с чем в ходе исследования анализу подлежали такие документы, как:

1) Регламент Европейского парламента и Совета № 1303/2013 от 17 декабря 2013 г. (далее - Регламент № 1303/2013), устанавливающий общие положения о Европейском фонде регионального развития, Европейском социальном фонде, Фонде сплочения, Европейском фонде развития сельских территорий и Европейском фонде морского судоходства и рыболовства [6];

2) Регламент Европейского Парламента и Совета ЕС № 1305/2013 от 17 декабря 2013 г. (далее - Регламент № 1305/2013), определяющий порядок поддержки сельских районов Европейским фондом развития сельских территорий (European Agricultural Fund for Rural Development - EAFRD) [7].

Для исследования вопросов создания, реализации и особенностей программы LEADER, причин еe расширения с помощью программы CLLD использовались необходимые архивные документы. В ходе изучения фактической информации, касающейся финансирования изучаемых программ, анализировались статистические данные Европейского фонда развития сельских территорий (EAFRD), демонстрирующие реальные расходы фонда непосредственно на мероприятия LEADER/CLLD [8]. Для рассмотрения организационных механизмов участия в программax LEADER/CLLD в исследовании привлекались соответствующие документы (в частности инструкции), изданные официальными органами ЕС [9], [10]. Обобщения и выводы, полученные в работе на основе скрупулезного изучения теории, законодательства и опыта реализации местных инициатив в границах сельских территорий, охватывают различные исследовательские проблемы, а именно: содержание обозначенных мер CAP (LEADER/CLLD) и специфичность их реализации на различных этапах реформирования политики; эффективность применения данных мер в современных условиях среды; целесообразность инкорпорации в отечественную политическую и хозяйственную практику мероприятий, основанных на непосредственном участии представителей сельских сообществ в разработке и реализации местных стратегий, в том числе с учетом существующих (глобальных, национальных) вызовов и угроз. 


\section{Agrarian Bulletin of the Urals No. 09 (212), 2021}

\section{Результаты (Results)}

Начать следует с уточнения некоторых значимых фактов. Во-первых, аббревиатура двух программ (подходов) используется в современных документах и публикациях как целостное обозначение (LEADER/ CLLD), однако их интерпретация и содержание все же несколько отличаются. Сокращение LEADER происходит от французской фразы Liaison Entre Actions De Développement De L'économie Rurale, что означает «Связь между различными видами деятельности для развития сельской экономики», а CLLD, в свою очередь, - от английского выражения CommunityLed Local Development, буквальный перевод которого звучит как «Руководимое сообществом местное развитие». Во-вторых, CLLD присоединился к LEADER относительно недавно (реформа САР 2013 г.), в то время как сама программа LEADER, являясь результатом предложений, выдвинутых в 1990 г. группой официальных лиц Европейской комиссии, принимается для реализации сначала в качестве эксперимента в 217 районах некоторых неблагополучных регионов ЕC (1991-1993 гг.), затем широко распространяется и активно реализуется во многих странах - членах ЕС (с 2000 г.), постепенно охватывая все типы сельских районов (2000-2006 гг.). Что касается более современных периодов развития LEADER, то следует выделить четвертый из них (2007-2013 гг.), в течение которого инициативы LEADER популяризируются, ими руководствуются уже 2416 сельских территорий во всех государствах - членах ЕС, их включают как обязательный компонент во все национальные программы развития сельских районов. Кроме того, в 2007 г. метод тематически расширяется и распространяется на политику в области рыболовства, в результате чего в этой отрасли в 21 государстве - члене ЕС создается более 300 местных инициативных групп. Примечателен следующий (пятый) период (20142020 гг.), в течение которого подход LEADER дополняется подходом CLLD [11], что означает, по сути, поддержку не только органичной взаимосвязи между отраслями и сферами деятельности сельской экономики, но и ее координацию местными сообществами. Причем если LEADER по-прежнему финансируется в рамках EAFRD, то CLLD пользуется финансовыми ресурсами и EAFRD, и других фондов, таких как Европейский фонд морского судоходства и рыболовства (EMFF), Европейский социальный фонд (ESF), Европейский фонд регионального развития (ERDF). Это, в свою очередь, позволяет местным инициативным группам реализовывать интегрированные стратегии развития сельских территорий, используя более широкие возможности (к их услугам сразу несколько фондов).

Обращаясь к содержательному аспекту подхода LEADER, важно, прежде всего, отметить семь его основных принципов.

Первый из них - планирование и реализация снизу вверх. Такой восходящий подход, закрепленный в нормативных актах ЕC, означает, что местное со- общество и местные «игроки» наиболее оптимальным образом определяют пути развития своего района, скрупулезно учитывая при этом потребности и ожидания населения. Взяв на себя ответственность за будущее территории (области, района, муниципалитета), они принимают решения относительно разработки местной стратегии сельского развития, ее реализации, анализа и оценки полученных результатов.

Второй приниип непосредственно связан с локальностью и территориальной привязкой. При «территориальном подходе» принятые программы нацелены на приоритеты территории в целом, учитывая, что она включает небольшую однородную, социально и функционально сплоченную локальность, характеризующуюся общими традициями, местной идентичностью, общими потребностями и ожиданиями. Такая сфокусированность на территории мотивирует местных жителей к сотрудничеству, выявляет сильные стороны, проблемы и возможности территориального развития, мобилизует для решения поставленных задач внутренний потенциал и человеческие, финансовые и экономические ресурсы того или иного сельского пространства. Как правило, охватываемая инициативами LEADER территория имеет четко определенные географические границы (обычно не совпадающие с административными границами) и соответствует обозначенным в статье 33 Регламента ЕС № 1303/2013 критериям по численности населения (10-150 тыс. человек), хотя государства - члены ЕС могут предъявлять к ним и дополнительные требования.

Третий приниип предполагает активное участие в развитии территорий «местных партнерств», которые институционализируются через специальный (структурированный) механизм управления - так называемую местную инициативную группу или местную группу действий (Local Action Groups - LAG). Участие в партнерстве означает, что движущими силами развития каждого территориального образования (области, района) являются представители местного населения, ставшие активными партнерами и инициирующие местное развитие. Согласно правилам ЕС, LAG должна включать представителей бизнеса, государственного и частного секторов общества, быть хорошо сбалансированной и отражать интересы различных социально-экономических групп на той или иной территории. В связи с этим на уровне принятия решений ни один сектор не может представлять более 49 \% членов местного партнерства (статья 32 Регламента № 1303/2013). Что касается юридической формы LAG, она может отличаться от страны к стране, но чаще всего такие местные инициативные группы регистрируются как некоммерческие организации.

В основу четвертого приниииа подхода LEADER и дополняющего его направления CLLD заложены интегрированные и многосекторальные стратегии местного развития. Следует подчеркнуть, что подход LEADER/CLLD именно этой позицией отличается от сугубо отраслевой (традиционной) сельскохозяй- 
|| ственной политики, реализуемой сверху вниз, нацеливаясь, таким образом, на активное использование связей между местными секторами экономики и опираясь на многочисленные мультипликативные эффекты такого взаимодействия. При этом интегрированность не означает всеобъемлющего характера, так как одни задачи лежат в пределах более узкого локального масштаба, другие же важны в границах той или иной LAG, а следовательно, все они имеют различный вес по значимости и подлежат решению в порядке их актуальности.

Пятый принциип LEADER сводится к важности в сельском развитии тесных сельских сетей, которые в значительной мере облегчают распространение необходимой информации, обмен знаниями, опытом, идеями, инновациями, ускоряют реализацию различных проектов развития сельских территорий, облегчают решение возникающих в современных (сложных, флуктуирующих) условиях проблем. Отмечая, что так называемый нетворкинг лежит в основе LEADER, способствуя установлению тесных связей между местными участниками и другими заинтересованными в успешном сельском развитии структурами, отметим, что сетевое взаимодействие в ходе реализации инициатив LEADER выходит далеко за рамки локального горизонта, в результате чего все более важными становятся (помимо местных) как национальные, международные сети, так и сети между сельскими и несельскими районами. Так, национальные сельские сети (NRN) созданы во всех государствах Европейского Союза в рамках программ развития сельских территорий, европейская же сеть развития сельских районов (ENRD) действует на общеевропейском уровне, представляя собой международную некоммерческую организацию, членами которой является большинство LAG стран - членов EC и многие добровольные национальные и региональные группы LEADER.

Стремление к инновациям можно идентифицировать как шестой принциип LEADER, отличающий данный подход с самого его основания. Участвуя в поиске и продвижении новых (инновационных) путей решения местных проблем, LEADER поддерживает на селе новые виды деятельности, производство новых продуктов или услуг, привнося всевозможные новшества в развитие определенной территории, создавая условия для роста добавленной стоимости в границах сельского пространства. Безусловно, не все в стратегии должно быть инновационным, поскольку многие из них направлены на удовлетворение определенных базовых потребностей. Однако более сложные задачи местные сообщества способны решать именно инновационным путем, по-новому используя потенциал имеющихся ресурсов, объединяя различные заинтересованные стороны и устанавливая диалог с внешними учреждениями, такими как университеты, исследовательские центры, администрации более высокие уровни. В лучшем случае партнерство может стать платформой и для «социальных иннова- ций», опыт и идеи которых могут быть проанализированы, задокументированы и переданы различным общеевропейским, национальным и региональным сетям участников LEADER/CLLD для имитации (использования). В целом же особенности инноваций в рамках LEADER/CLLD следующие: могут включать новые продукты, услуги или способы ведения определенной деятельности в местном контексте; часто имеют мультипликативный эффект от изменений, предпринятых сообществом; включают в себя одно или несколько небольших мероприятий или крупномасштабных проектов, мобилизующих сообщество на динамичное развитие; означают новые способы аккумулирования и использования существующих ресурсов и активов сообщества; стимулируют сельское сообщество к сотрудничеству между различными участниками и секторами.

И наконец, седьмой принщип, который является продолжением всех предыдущих и предполагает открытость к сотрудничеству по всем направлениям и во всех его проявлениях. Возможности местного развития в сельских районах расширяет, как правило, межтерриториальное и международное сотрудничество. Вовлекая местных жителей и местные LAG в совместную работу над различными совместными проектами, сети могут включать другие группы LEADER, сформированные в других регионах, государствах - членах ЕС или даже в странах, не входящих в ЕС, но имеющих прочные традиции местного самоуправления и сетевого взаимодействия.

Сотрудничество с другими субъектами при этом может:

1) служить источником инноваций и каналом передачи знаний для местного населения;

2) привносить в локальное развитие идеи из внешней для него среды (другие территории, сферы деятельности);

3) импортировать и экспортировать успешные практики и передовой опыт.

Исторически механизмы реализации подхода LEADER, модифицируясь на различных этапах Единой сельскохозяйственной политики ЕС, несколько отличаются и на современных этапах реформ САР. Прежде всего, доказав свою эффективность в содействии развитию сельских районов за счет полного учета межотраслевых потребностей в эндогенном сельском развитии, LEADER признан целесообразным для его использования в будущем, вследствие чего его применение остается обязательным для программ развития сельских районов на национальном и региональном уровнях. Особенности осуществления мероприятий LEADER в период 2014-2020 гг. определяются Регламентом № 1303/2013 и Регламентом № 1305/2013, причем, чтобы дать возможность партнерам в сельской местности протестировать и подготовиться к разработке и реализации стратегии местного развития (тем, кто ранее не участвовал в подобных мероприятиях), предусмотрено финансирование «Стартового комплекта LEADER». Важно 


\section{Agrarian Bulletin of the Urals No. 09 (212), 2021}

подчеркнуть, что мероприятия LEADER согласуются практически со всеми тематиками национальных программ стран - членов EC, а такая мера, как «Поддержка местного развития LEADER - развитие под руководством местных сообществ», предусмотренная в статье 35 Регламента № 1303/2013 и обозначенная в Регламенте № 1305/2013 как «Мера 19», применима практически ко всем фокус-группам, сформулированным в содержании Единой сельскохозяйственной политики ЕС.

Относительно финансирования инициатив LEADER ситуация такова, что максимальный вклад Европейского фонда развития сельских территорий (EAFRD) составляет 80 \% для мер, указанных:

1) в статьях 14 (трансфер знаний и инноваций);

2) пункте (a/i) статьи 19.1 (поддержка молодых фермеров);

3) в статье 27 (создание групп и организаций производителей);

4) в статье 35 (кооперация) Регламента № $1305 / 2013$;

5) в статье 32 Регламента (ЕС) № 1303/2013 (местное развитие под руководством сельских сообществ).

Эта ставка может быть увеличена максимум до $90 \%$ для аналогичных программ в менее развитых или наиболее удаленных регионах. Общая сумма, делегированная EAFRD на выполнение инициатив LEADER, составляет на 2014-2020 гг., согласно плану CAP, 7011,4 млн евро. В соответствии с отчетной информацией фонда за 2020 г. (включает данные и за предыдущие годы) расходы на LEADER составили 2770,4 млн евро.

Если обратиться к механизмам финансирования LEADER в ретроспективе, то можно констатировать следующее:

1) в период 1991-1993 гг. объем финансирования составил 450 млн евро, финансирование осуществлялось через несколько фондов (основным выступил Европейский сельскохозяйственный консультационный и гарантийный фонд - EAGGF, дополнительные средства предоставляли Европейский социальный фонд - ESF и Европейский фонд регионального развития - ERDF), в реализации политики участвовало 217 местных инициативных групп (LAG);

2) в период 1994-1999 гг. объем финансирования - 1,7 млрд евро, каналы финансирования прежние (EAGGF, ESF, ERDF), приняли участие 821 LAG;

3) в период 2000-2006 гг. объем финансирования 2,1 млрд евро, институтом финансирования выступил EAGGF, участвовало 1143 местные инициативные группы (893 из состава EU-15 и 250 - из состава шести стран, вновь вошедших в состав ЕС);

4) в период 2007-2013 гг. финансирование осуществлялось через Европейский фонд развития сельских территорий (EAFRD), объем финансирования возрос до 5,5 млрд евро (6 \% бюджета EAFRD), участвовало 2200 инициативных групп;

5) в период 2014-2020 гг. (LEADER/CLLD) финансирование предусматривалось через группу фон- дов ESI (Европейские структурные и инвестиционные фонды, которые объединяют пять фондов, такие как отмеченные ранее EAFRD, ESF, ERDF и Фонд сплочения (CF), а также Европейский фонд морского судоходства и рыболовства (EMFF)), участие приняло более 3000 местных инициативных групп, в структуpe расходов EAFRD поддержка мер LEADER составила 4,5 \% вместо 7 \% запланированных [12].

Современный этап развития LEADER, когда к еe основным принципам и подходам добавились механизмы CLLD, заслуживает особого внимания и пристального изучения. Как показывает статистика, применение принципов, лежащих в основе LEADER и CLLD, распространилось за время их использования от небольшого кластера из нескольких пилотных проектов до множества практик более 3000 партнерств, функционирующих почти во всех уголках сельской Европы. Так, если учитывать условия софинансирования рассматриваемых инициатив, общие государственные и частные инвестиции, осваиваемые этими партнерствами в целях улучшения занятости населения, предоставления местных услуг и защиты окружающей среды, в период 2007-2013 гг. составили примерно 8,6 млрд евро. Основное преимущество LEADER/CLLD, по мнению аналитиков, заключается в том, что к управлению сельским развитием привлекаются люди, испытывающие значимые потребности и специфические проблемы. Пути их удовлетворения и преодоления определяются местными сообществами, а люди (местное население), которые ранее были пассивными «бенефициарами», становятся активными партнерами и движущей силой развития соответствующих территорий.

В итоге стратегии LEADER/CLLD, проистекая из конкретных вопросов или проблем, волнующих местное сообщество (к примеру, упадок традиционных отраслей экономики, неудовлетворенность сельскими условиями жизни молодежи и сельского населения в целом, негативные следствия изменения климата), выстраиваются на связях между секторами и людьми таким образом, что генерируется положительный мультипликативный эффект, улучшающий результаты национальных и региональных программ развития сельских территорий, позволяющий решать множество проблем в их местном контексте, мобилизовать все соответствующие политики и ресурсы, укрепляя взаимодействие между:

1) различными местными департаментами, муниципалитетами и государственными администрациями;

2) местными общественными и частными организациями (организациями гражданского общества);

3) учреждениями местного и более высокого уровня, такими как региональные и национальные правительства, университеты, другие элементы местных кластеров.

Из опыта реализации подходов LEADER/CLLD в государствах - членах EC можно сделать не только имеющие значение для применения в других странах 
|| теоретические заключения о важности использования местного потенциала (ресурсов, сетей и т. д.) в сельском развитии, но и совершенно конкретные прикладные выводы, инкорпорация которых в политическую и хозяйственную практику непременно принесет положительные результаты в решении проблем развития сельских территорий. Прежде всего, они целесообразны для учета на начальных этапах формирования активных LAG.

1. Время и ресурсы, необходимые для подготовки к реализации принципов LEADER/CLLD в той или иной стране (в границах определенной территории), во многом зависят от местного контекста, главным образом от практического опыта и способности местных участников (частных лиц, организаций) реализовывать совместные проекты, их материальных и нематериальных возможностей. Как показывают аналитические данные, даже в опытных сообществах на подготовку к активному участию в LEADER/CLLD обычно уходит от шести месяцев до года. С одной стороны, это процесс, не имеющий единого алгоритма в силу разнообразия местных сообществ. С другой стороны, такое разнообразие приносит множество новых идей, раскрывает наличие новых ресурсов и возможностей, имеющих решающее значение для будущего успеха.

2. Запуск самого процесса инициирования и реализации мероприятий CLLD можно разбить на серию итерационных шагов или циклов, вписывающихся в три основных компонента (стратегия, партнерство, сфера реализации) и представляющих собой движение по спирали (с возвращением к тому или иному сегменту на более высоком уровне) [10, с. 14].

Первый шаг сводится к выявлению того, что необходимо изменить в определенном территориальном пространстве (сегмент «стратегия»). В данном отношении CLLD-подход модифицирует традиционный порядок определения стратегии развития (сверху вниз), начиная ее спецификацию с видения того, что местные жители хотят иметь и что, по их мнению, следует изменить, чтобы достичь этого. На первый план выступает, как правило, формирование целей в соответствии с местными потребностями, а финансирование рассматривается как средство их достижения. Таким образом, формулирование четкого понимания того, что необходимо изменить в границах определенной локальности, - первый и самый важный шаг в разработке стратегии.

Второй шаг заключается в создании доверительных отношений между людьми, которые могут помочь достичь желаемых изменений (сегмент «партнерство»). Этому может помочь использование формальных инструментов, в том числе исследовательских, таких, к примеру, как «анализ заинтересованных сторон», определяющий и характеристики различных бенефициаров, и уровень их заинтересованности, и способность влиять на процесс реализации CLLD. Этот шаг обычно происходит параллельно с принятием решения о том, что сообщество хочет изменить.
Для этого требуются личный контакт и время, чтобы выявить и обсудить скрытые проблемы и недовольства, которые, возможно, исторически глубоко укоренились в жизни сообществ. Индивидуальные и групповые обсуждения при этом могут помочь прояснить как долгосрочные цели, так и возможные краткосрочные действия, которые способны привести к более эффективным результатам. Прежде чем создать официальную (формальную) партнерскую структуру, полезно укрепить доверие и получить опыт совместной работы с помощью неформального тесного общения.

Третий шаг предполагает определение границ территории, участвующей в реализации принципов LEADER/CLLD (сегмент «сфера реализации»), причем, во-первых, они не совпадают с границами административными, во-вторых, должны строго соответствовать национальным или региональным критериям, в широких рамках которых местные участники определяют наиболее подходящее пространство для достижения своих целей. Безусловно, территория, с одной стороны, должна быть достаточно большой, чтобы, как говорится в документах CLLD, иметь необходимую «критическую массу» для достижения своих целей, с другой стороны, она не может быть слишком большой, чтобы не возникало рисков, обусловленных потерей контроля со стороны сообщества.

Четвертый шаг означает непосредственную подготовку местной стратегии развития, основанную на потребностях местного населения и активном его вовлечении в ее реализацию (снова сегмент «стратегия»). Как только достигается широкое согласие относительно того, что сообщество хочет изменить и кто может помочь в достижении этого, и уточняется область вмешательства, на повестку дня выносится точная спецификация деталей местной стратегии, разработка которой основана на глубоком SWOT-анализе (анализе сильных и слабых сторон, возможностей и угроз, с которыми сталкивается территория). Стратегия местного развития становится, по сути, дорожной картой внедрения CLLD, согласно которой местные инициативные группы (партнерства) выбирают и поддерживают проекты, ранжируя их значимость в соответствии с их вкладом в достижение целей стратегии.

По поводу особенностей анализа необходимо отметить следующее:

1) вопросы, поднятые в SWOT-анализе, должны основываться на данных, полученных в результате тщательной диагностики местности;

2) анализ необходимо сосредоточить на конкретных особенностях района и причинах его уникальности, а не просто на перечислении характеристик ведущих секторов экономики (сельского хозяйства, рыболовства, к примеру) или целевых групп (молодежи, женщин);

3) больше внимания следует уделять картированию возможностей и местных «активов» (помимо описания слабых сторон и угроз); 


\section{Agrarian Bulletin of the Urals No. 09 (212), 2021}

4) важно сформировать дифференцированный список задач, ранжированный согласно приоритизации потребностей и с учетом имеющегося потенциала.

Пятый шаг вновь обращается к партнерству, а именно к уточнению его структуры и определению того, кто и чем именно будет заниматься в ходе реализации стратегии (сегмент «партнерство»). Следует отметить, что местные сообщества обладают разными уровнями возможностей, индивидуальным опытом совместной работы, специфической институциональной культурой, в связи с чем важной частью подготовительной работы является такое структурирование партнерства, которое наилучшим образом соответствует местным реалиям и локальному контексту. Партнерства при этом (чаще всего) придерживаются двух общепринятых в ЕС моделей, первая из которых предполагает создание совершенно нового (объединяющего местных участников) юридического лица, которое может принимать различные организационные формы, однако предпочтение отдается в первую очередь некоммерческой организации (ассоциации, к примеру). Независимо от принятой правовой формы организация должна широко представлять местные заинтересованные стороны, быть открытой, прозрачной и подотчетной как местным жителям, так и своим спонсорам. Точный баланс вовлеченных партнеров и степень их участия в принятии решений зависят от местных условий, но, как упоминалось ранее, ключевой особенностью CLLD является то, что в партнерских отношениях не должна доминировать какая-либо отдельная группа, представляющая как общественные, так и частные интересы.

Вторую модель можно использовать, когда нет необходимости создавать дополнительную структуру или когда более выгодно использовать административные возможности одного из опытных партнеров. В этом случае он становится «подотчетным органом» с юридической и административной точек зрения, в то время как другие партнеры образуют своего рода комитет по принятию решений и отбору проектов, причем принципы репрезентативности, открытости, подотчетности и прозрачности остаются обязательными к применению, как и в первой модели. Важно, что обе представленные модели могут использоваться для координации нескольких потоков финансирования.

Шестой шаг, следуя спиралевидному алгоритму реализации принципов LEADER/CLLD, сводится опять же к регулированию границ применения уточненной стратегии сельского развития (сегмент «сфеpa реализации»). Так, в процессе подготовки стратегии местного развития и установления партнерских отношений часто становится очевидным, что определенные проблемы можно лучше решить, учитывая возможности других (прилегающих к сельским) территорий, в частности близлежащих (городских, районных) локальностей, обеспечивающих рабочие места и предоставляющих те или иные услуги.
В результате потенциально полезными становятся территории, лежащие за пределами первоначально определенных границ. Кроме того, с одной стороны, улучшение синергии между местными инициативами, финансируемыми различными фондами ЕС, иногда возможно лишь путем изменения предварительно определенных границ, с другой стороны, национальные и региональные критерии выбора зон для CLLD могут потребовать некоторой корректировки в определении границ партнерства.

Таким образом, точные границы вмешательства рассматриваются подходами LEADER/CLLD как нечто подвижное, адаптируемое к меняющимся обстоятельствам, a CLLD предлагает местным партнерам ряд альтернатив для решения проблем различного масштаба или так называемое гибкое «меню» для обеспечения того, чтобы границы соответствовали меняющимся местным потребностям, а не фиксировались в определенный момент времени. Например, соседние местные партнерства могут непосредственно сосредоточиться на действиях, финансируемых одним конкретным фондом, но затем использовать соответствующие меры для сотрудничества или совместно участвовать в мероприятиях, финансируемых другим фондом, для решения проблем, которые лучше всего решаются на ином, трансграничном уровне. Важно иметь в виду, что расширение границ не должно приводить к потере чувства местной идентичности и сокращению реального участия местных сообществ в реализации мероприятий LEADER/CLLD.

Седьмой шаг, приближающий стратегию к ее успешной реализации, заключается в спецификации подробного плана действий и подготовке заявки на финансирование (еще раз сегмент «стратегия»). По сути, после того как партнерством окончательно согласованы вопросы, что именно необходимо изменить, какова логика действия, составляется реалистичный план, на основании которого формируется заявка на финансирование. Обоснованный таким образом план должен быть, с одной стороны, детальным и адекватным (реалистичным), с другой же - обладать определенной гибкостью для реагирования на непредвиденные обстоятельства. Государства - члены EC, отдельные регионы и местные партнерства решают эту проблему по-разному, однако в любом случае для получения финансирования важно показать, что график реализации, выделенные человеческие, материальные и финансовые ресурсы соответствуют выявленным ранее потребностям и имеют разумные шансы на достижение желаемых изменений. Более того, партнерство должно продемонстрировать, что у него есть навыки, опыт и отработанные процедуры, гарантирующие реализацию плана эффективным и прозрачным образом.

В заключение, восьмой шаг, как это принято при осуществлении той или иной политики, предусматривает создание целостной сложной системы периодического анализа, оценок и обновления стратегии. В связи с этим в обзоре мероприятий LEADER/CLLD 
Европейская аудиторская палата утверждает, что мониторинг, самооценка и внешняя оценка местных стратегий развития нуждаются в постоянном совершенствовании [13].

Если обратиться к реальным ситуациям, с которыми столкнулись местные сообщества в программном периоде 2014-2020 гг., кардинально отличающемся от двух предыдущих периодов, можно заметить, что они испытали все сложности экономического кризиса, были вынуждены искать дополнительные источники средств для элементарного выживания, осознали необходимость решения многих обострившихся социальных, экологических и экономических проблем для обеспечения долгосрочной жизнеспособности многих местных сообществ [14]. Изменения во внешнем контексте, а именно долгосрочные глобальные проблемы, с которыми сталкивается Европа, включая снижение конкурентоспособности на фоне успехов развивающихся экономик, глобальное потепление и истощение ресурсов, стагнацию производства и снижение реальной заработной платы, падение уровня жизни значительной части населения(в том числе сельского), растущее неравенство и социальную поляризацию, проблемы в системах здравоохранения и социального обеспечения (отчасти вызванные старением населения и другими неблагоприятными демографическими изменениями), затрудняют поиск универсальных решений на уровне ЕС и все более основательно аргументируют важность поиска подходов, адаптированных к местным условиям.

На фоне глобальных проблем местный контекст и локальные потребности людей претерпевают глубокие изменения, в результате чего становится очевидной необходимость использования обществом различных (нетрадиционных, инициируемых и реализуемых снизу) практик, например, социально и территориально укоренившихся форм предпринимательства, различных форм самопомощи, коллективных форм мобилизации активов сообществ, мер реагирования на изменение климата (его последствия) на местном уровне.

Уже к началу программного периода 2014-2020 гг., а особенно сейчас, в эпоху пандемии COVID-19, в Европе отмечаются такие неблагоприятные условия, как:

1) рост безработицы, особенно среди молодежи, причем политики расценивают это как риск утраты в границах сельских территорий высокообразованного поколения и увеличения количества недовольных молодых людей [10, с. 20];

2) сокращение во многих странах внутреннего потребления (следовательно, и спроса), что обуславливает тем самым ухудшение ситуации во многих отраслях производства, в то время как сельское хозяйство, рыболовство и пищевая промышленность отличаются относительной стабильностью даже в тяжелые времена коронакризиса и заслуживают пересмотра их роли в местной экономике;
3) сокращение частного финансирования сельского развития из-за отвлечения ресурсов на стратегически важные направления развития бизнеса, что детерминирует поиск доступа к альтернативным источникам финансирования;

4) уменьшение государственных инвестиций в развитие сельских территорий в силу расширения спектра насущных потребностей в государственной поддержке по различным экономическим, социальным и экологическим направлениям, что затрудняет (как следствие) поиск государственного софинансирования сельских проектов;

5) неадекватность сельской инфраструктуры во многих государствах - новых членах ЕС, что является, по сути, серьезным препятствием для местного развития;

6) существенное секвестирование во многих странах расходов на образование, здравоохранение, социальные услуги, в связи с чем местные власти активно используют средства фондов ЕС для предоставления основных услуг на селе;

7) рост в большинстве стран (особенно на фоне COVID-19) бедности и социальной изоляции, порождающих множество новых трудно решаемых задач;

8) необходимость (в ответ на имеющие место изменения климата) перехода к низкоуглеродной экономике (необходимость сокращения выбросов парниковых газов, важность формулирования новой парадигмы экономического развития, основанной на концепции «зеленой» экономики и «зеленого» роста, предельная актуальность устойчивого использования ресурсов).

В ответ на эти сложности большинство проектов LEADER/CLLD, во-первых, являются инновационными с точки зрения подходов, организации, финансирования и других характеристик, во-вторых, охватывают расширение и улучшение разнообразных сфер сельской жизни. Кроме того, основные направления проектов включают развитие предпринимательства и сельского туризма, увеличение числа рабочих мест, обеспечение доступа к широкополосному интернету, социальную интеграцию, базовые услуги, предназначенные для труднодоступных сообществ, сельской молодежи, сельской среды, охрану и рациональное использование водных ресурсов, защиту и улучшение биоразнообразия, развитие возобновляемой энергетики. В целом же особенность подхода LEADER/CLLD заключается в постепенном переходе от простых информационных кампаний и официальных консультаций к реальному партнерству и практически полному контролю граждан за реализацией инициированных ими проектов.

Обсуждение и выводы (Discussion and Conclusion)

Основываясь на обзоре проводимых для продвижения LEADER/CLLD мероприятий, следует отметить, что опыт реализации данного подхода в государствах - членах ЕС вызывает интерес и в самих европейских странах, и в мире в целом. Как следствие, на всевозможных научных и прикладных дис- 


\section{Agrarian Bulletin of the Urals No. 09 (212), 2021}

куссионных площадках обсуждаются результаты осуществления соответствующих локальных проектов и местных инициатив, а также перспективы развития LEADER/CLLD в течение нового программного периода САР (2021-2027 гг.). Тематика обсуждения охватывает разнообразные вопросы: от создания умных деревень и вовлечения молодежи в развитие сельских территорий до применения временных инструментов восстановления сельских территорий после экономического и социального ущерба, нанесенного пандемией COVID-19.

В теоретическом отношении рассмотрению подлежат новые практики развития сельских районов в рамках LEADER/CLLD, которые можно охарактеризовать как неоэндогенные [15, с. 357], основанные на идее о том, что социально-экономическое благополучие территорий может быть достигнуто более успешно путем привлечения к управлению территориальным развитием локальных межотраслевых партнерств, которые лучше учитывают местные условия и потребности [16]. В практическом плане актуальными становятся вопросы о том, как в контексте LEADER/CLLD обеспечить гибкость и адаптируемость проектов к возникающим на сельских территориях вызовам, связанным с последствиями изменений климата, пандемией COVID-19, депопуляцией и старением населения в сельских районах. Как итог - гибкость, релятивность, устойчивость, укрепление сетей и сотрудничества - аспекты LEADER/CLLD, идентифицируемые в качестве предельно важных и отдельными сельскими сообществами на локальном уровне, и государствами - членами ЕС в более широком контексте. «Социальная устойчивость», основанная на наращивании особого человеческого и социального капитала, социальных инновациях и социальной интеграции, расценивается научным сообществом и практиками в качестве одной из основных целей программ LEADER [17].

Длительный опыт реализации LEADER позволил ученым и практикам обобщить имеющуюся информацию о ходе реализации программ в странах - членах ЕС и проанализировать имеющиеся в них примеры применения подхода LEADER к местному развитию [18-20]. Как показывает обзорно-исторический анализ, главная идея развития сельских территорий на основе инициатив LEADER состоит в том, что местные ресурсы, местное сотрудничество и местные сети имеют решающее значение для продвижения локального развития и социальных инноваций, составляя при этом существенный потенциал развития. Используя такие методы, как экспертные мнения заинтересованных сторон, построение «деревьев проблем», анализ сценариев, местные инициативные группы (LAG) специфицируют проблемы, которые действительно важны для людей на местах, мобилизуют их идеи и энергию, формулируют стратегические документы, позволяющие получить поддержку и финансирование в рамках САР. Ключевым драйвером сельского развития в русле LEADER/CLLD являются общие ценности, общее управление, общие ресурсы.

Резюмирующие положения, сформулированные в данной статье, можно использовать для разработки научно-практических рекомендаций относительно того, как европейский опыт реализации LEADER/ CLLD следует применять в качестве конструктивного метода развития сельских территорий в России. В качестве основных аргументов, обосновывающих полезность данного опыта для мировой (в том числе отечественной) практики сельского развития, можно выдвинуть следующие:

1. Высокую научно-практическую значимость имеет сама идея использования подхода LEADER/ CLLD в развитии сельских территорий, что означает:

1) опору на потенциал сообществ при поддержке государства (а не наоборот), что дает возможность оптимально расходовать средства и эффективно выполнять задачи сельского развития;

2) наиболее точное определение проблем развития сельских территорий в той или иной локальности, их ранжирование по приоритетам местного населения;

3) возможность гибко и своевременно адаптироваться к изменениям среды жизнедеятельности сельских сообществ, а следовательно, изменениям их нужд и преференций.

2. Инкорпорации в политическую практику заслуживают положительно зарекомендовавшие себя в течение 30 лет механизмы реализации LEADER (a сегодня за период 2014-2020 гг. и CLLD), а именно:

1) порядок создания местных инициативных групп (LAG), выстраивание структуры управления и функционирования;

2) опыт взаимодействия LAG с финансирующими их работу фондами;

3) положительные практики осуществления местных проектов в различных сельских условиях (удаленные сельские территории, горные районы, обезлюдевшие (с малочисленным сельским населением) местности, имеющие слабую инфраструктуру муниципалитеты).

3. Проблемно ориентированный анализ реализации подхода LEADER/CLLD в границах Европейского союза высвечивает очевидное смещение вектора приоритетов европейских LAG в сторону:

1) обновленных социальных требований сельского населения (современная инфраструктура, универсальный и недорогой доступ к высокоскоростной широкополосной связи, устойчивая мультимодальная мобильность для сельской местности);

2) проблем, связанных с изменением климата, следствия которого все более жестко сказываются на условиях жизни селян (неблагоприятные изменения условий ведения аграрной деятельности, погодные катаклизмы, ухудшение качества почвы, водных ресурсов и т. д.);

3) эколого-охранных акций как важной предпосылки сохранения сельских территорий.

4. Интерес представляют не только приоритеты, механизмы и инструменты реализации LEADER/ 
CLLD странах-членах EC, но и конкретные мероприятия, инициируемые LAG непосредственно в сегодняшних обстоятельствах. К таковым следует отнести:

1) создание платформы возрождения села - информационно-консультационной площадки для тесного сотрудничества представителей местных сообществ в процессе решения самых разнообразных их проблем;

2) развитие исследований и инноваций для сельских сообществ;

3) поддержка образования и профессионального обучения, спорта и волонтерской деятельности в сельской местности;

4) расширение участия в программе Smart Villages;

5) продвижение лучших практик мультимодальной мобильности для сельской местности;

6) продвижение цифрового будущего для сельских районов;

7) повышение доступности сельских районов с помощью Drone Strategy 2.0;

8) поддержка сельских муниципалитетов в переходе к новым источникам энергии и в борьбе с изменением климата;

9) улучшение качества почв, воды и воздуха;

10) обеспечение социальной устойчивости, в том числе за счет улучшения положения женщин в сельской местности; тей;

11) гарантия на селе равных возможностей для де-

12) продвижение предпринимательства и социальной экономики в сельской местности;

13) содействие развитию устойчивой биоэкономики;

14) усиление роли кооперации (сетей) производителей и сотрудничества сельских жителей в развитии сельских территорий;

15) расширение возможностей образования, профессиональной подготовки и трудоустройства молодых людей в сельских (особенно отдаленных) районах.

Перечень возможностей использования европейского опыта государственной поддержки сельских территорий, в том числе основанного на активном вовлечении в социально-экономические и экологические процессы местного (сельского) населения, можно перманентно расширять по мере его накопления, совершенствования и обобщения. В целом же отметим, что бо́льшая часть приоритетов САР на последние программные периоды ее реализации (20142020 гг., 2021-2027 гг.) имеет высокую актуальность для отечественной политики в области развития сельских территорий, а механизмы и инструменты их реализации через LEADER/CLLD, безусловно, полезны для использования в политической и хозяйственной практике.

\section{Благодарности (Acknowledgements)}

Исследование выполнено при финансовой поддержке РФФИ в рамках научного проекта № 19-2907315.

\section{Библиографический список (References)}

1. Abdullah A., Khadaroo I. The Trust-Control Nexus in Public Private Partnership (PPP) contracts // The Journal of Accounting and Public Policy. 2020. No. 39 (6). Article number 106768.

2. Dupraz P., Guyomard H. Environment and Climate in the Common Agricultural Policy // EuroChoices. 2019. Vol. 18. No. 1. Pp. 18-25.

3. Roth S., Valentinov V., Kaivo-oja J., Dana L.-P. Multifunctional Organisation Models // Journal of Organizational Change Management. 2018. No. 31 (7). Pp. 1383-1400.

4. Cheng Z., Wang H., Xiong W., Zhu D., Cheng L. Public-Private Partnership as a Driver of Sustainable Development: Toward a Conceptual Framework of Sustainability-Oriented PPP // Environment, Development and Sustainability. 2021. No. 23. Pp. 1043-1063.

5. Pawłowska A. Territorial Partnerships in Rural Regions - Neo-Institutional Perspective // Polish Sociological Review. 2017. No. 1. Pp. 95-108.

6. European Parliament. Regulation (EU) No 1303/2013 of The European Parliament and of the Council of 17 December 2013 Laying Down Common Provisions on the European Regional Development Fund, the European Social Fund, the Cohesion Fund, the European Agricultural Fund for Rural Development and the European Maritime and Fisheries Fund and Laying Down General Provisions on the European Regional Development Fund, the European Social Fund, the Cohesion Fund and the European Maritime and Fisheries Fund and repealing Council Regulation (EC) No 1083/2006 // Official Journal of the European Union. 2013. Vol. 56. No. 347. Pp. 320-469.

7. European Parliament. Regulation (EU) No 1305/2013 of The European Parliament and of the Council of 17 December 2013 on Support for Rural Development by the European Agricultural Fund for Rural Development (EAFRD) and Repealing Council Regulation (EC) No 1698/2005 // Official Journal of the European Union. 2013. Vol. 56. No. 347. Pp. 487-548.

8. Report from the Commission to the European Parliament and the Council 14th Financial Report from the Commission to the European Parliament and the Council on the European Agricultural Fund for Rural Development (EAFRD) 2020 Financial Year [e-resource]. URL: https://eur-lex.europa.eu/legal-content/EN/TXT/?uri=CELEX:52021DC0539 (date of reference: 18.07.2021). 
9. Guidance on Community-Led Local Development for Local Actors. 2014-2020 [e-resource]. URL: http:// ec.europa.eu/regional_policy/sources/docgener/informat/2014/guidance_clld_local_actors.pdf (date of reference: 18.07.2021).

10. Guidance on Community-led Local Development in European Structural and Investment Funds (version from June 2014) [e-resource]. URL: http://ec.europa.eu/regional_policy/sources/docgener/informat/2014/guidance_co mmunity_local_development.pdf (date of reference: 08.07.2021).

11. Servillo L., De Bruijn M. From LEADER to CLLD: the Adoption of the New Fund Opportunities and of their Local Development Options // European Structural and Investment Funds Journal. 2018. No. 6. Pp. $223-233$.

12. Roncevic A., Lez T., Gregoric M. Implementation of the Leader Programme in the Observed EU Countries. Economic and Social Development (Book of Proceedings), 50-th International Scientific Conference on Economic and Social Development Development, 2020. Pp. 99-107.

13. Kokot J. ECA Audits of the CAP Highlight Issues that Tie into the Recent Agreement on the Future of the CAP // ECA Journal. 2021. No. 2. Pp. 61-66.

14. Wang H., Xiong W., Wu G., Zhu D. Public-Private Partnership in Public Administration Discipline: A Literature Review // Public Management Review. 2018. No. 20. Pp. 293-316.

15. Furmankiewicz M., Janc K, Walsh A. M. Implementation of the EU LEADER Programme at Member-State Level: Written and Unwritten Rules of Local Project Selection in Rural Poland // Journal of Rural Studies. 2021. Vol. 86. Pp. 357-365.

16. Salemink K., Strijker D., Bosworth G. The Community Reclaims Control? Learning Experiences from Rural Broadband Initiatives in the Netherlands // Sociologia Ruralis. 2017. No. 57 (S1). Pp. 555-575.

17. Mohd Som R., Omar Z., Ismail I. A., Alias S. N. Understanding Leadership Roles and Competencies for PublicPrivate PartnerShip // Journal of Asia Business Studies. 2020. No.14. Pp. 541-560.

18. Ballesteros J. G. T., Hernández M. Promoting Tourism through the EU LEADER Programme: Understanding Local Action Group Governance // European Planning Studies. 2019. No. 27 (2). Pp. 396-414.

19. Konecný O. The Leader Approach Across the European Union: One Method of Rural Development, Many Forms of Implementation // European Countrys. 2019. No. 11. Pp. 1-16.

20. Neumeier S. Social Innovation in Rural Development: Identifying the Key Factors of Success // Geographical Journal. 2017. No. 183. Pp. 34-46.

\title{
Об авторах:
}

Светлана Георгиевна Головина ${ }^{1,3}$, доктор экономических наук, профессор, главный научный сотрудник института аграрно-экологических проблем и управления сельским хозяйством, ORCID 0000-0002-1157-8487, AuthorID 149863; +7 909 146-40-64,s_golovina@yahoo.com

Алексей Владимирович Ручкин ${ }^{1}$, кандидат социологических наук, доцент, доцент кафедры менеджмента и экономической теории, ORCID 0000-0002-6981-3080, AuthorID 615361; + 7909 022-78-24,

alexeyruchkin87@gmail.com

Иван Николаевич Миколайчик 2 , доктор сельскохозяйственных наук, профессор, проректор по научной работе, ORCID 0000-0001-5189-2174, AuthorID 149861; +7912 522-64-64,min_ksaa@mail.ru

Лидия Николаевна Смирнова ${ }^{2}$, кандидат педагогический наук, ведущий научный сотрудник НИИ «Изучение проблем АПК», ORCID 0000-0002-7991-0802, AuthorID 904486; +7 912 212-68-17, lidia-1311@mail.ru

${ }^{1}$ Уральский государственный аграрный университет, Екатеринбург, Россия

${ }^{2}$ Курганская государственная сельскохозяйственная академия имени Т. С. Мальцева, Лесниково, Россия

${ }^{3}$ Российский государственный аграрный заочный университет, Москва, Россия

\section{Local communities participation in rural development: the experience of the European Union}

\author{
S. G. Golovina ${ }^{1,3 凶}$, A. V. Ruchkin ${ }^{1}$, I. N. Mikolaychik ${ }^{2}$, L. N. Smirnova ${ }^{2}$ \\ ${ }^{1}$ Ural State Agrarian University, Ekaterinburg, Russia \\ ${ }^{2}$ Kurgan State Agricultural Academy, Lesnikovo, Russia \\ ${ }^{3}$ Russian State Agrarian Correspondence University, Moscow, Russia \\ E-mail:s_golovina@yahoo.com
}

Abstract. The use of the experience of implementing the Common Agricultural Policy (CAP) in the member states of the European Union (EU) is relevant both for Russia and for other countries of the world interested in the successful development of the agrarian sector of the economy and rural areas. The role of rural areas in achieving national secu- 
rity of countries and regions (food, biological, environmental and other) is increasing significantly due to the current challenges and threats (climate change, COVID-19 pandemic, aggravation of the international situation). The purpose of the study, the results of which are presented in this article, is to scrutinise the special approach implemented under the CAP. This approach is referred to in European law as LEADER/CLLD and refers to a close combination of comprehensive cross-sector interaction with active involvement of local communities in rural development. In the work, analytical and review research methods were used, with the help of which (1) the current (relevant to the research topic) legislation, (2) programs implemented in the EU member states, (3) significant scientific publications were subject to scrupulous study. The result of the work is a review and analysis of the findings and practical recommendations for the future use of the various aspects of LEADER/CLLD in domestic political and economic practice. The application of this approach takes into account the fact that the experience of local residents, combined with the opinions of other stakeholders, can help to better adapt rural development policy to real needs and opportunities, and to form a specific (unique) human capital within the boundaries of rural communities. Human capital includes, in addition to specific skills, (1) the ability to take constructive initiatives, (2) a sense of local identity and ownership, (3) the ability to participate as equals with other partners in defining local development strategies, (4) trust between people, private enterprises, public institutions and sectoral communities interested in successful rural development, formed through constant interaction. Theoretical and practical conclusions regarding the content of LEADER/CLLD initiatives, as well as findings related to the possibilities of introducing tools and mechanisms to support rural areas, implemented directly with the involvement of local communities with financial support from the state, are of scientific novelty.

Keywords: Common Agricultural Policy, European Union, rural areas, LEADER/CLLD, challenges and threats.

For citation: Golovina S. G., Ruchkin A. V., Mikolaychik I. N., Smirnova L. N. Uchastie mestnykh soobshchestv v razvitii selэskikh territoriy: opyt Evropeyskogo soyuza [Local communities participation in rural development: the experience of the European Union] // Agrarian Bulletin of the Urals. 2021. No. 09 (212). Pp. 80-92. DOI: 10.32417/1997-4868-2021-212-09-80-92. (In Russian.)

Date of paper submission: 26.07.2021, date of review: 03.08.2021, date of acceptance: 09.08.2021.

\section{Authors' information:}

Svetlana G. Golovina ${ }^{1,3}$, doctor of economics, professor, chief researcher of the research institutes of agricultural and environmental problems and agricultural management, ORCID 0000-0002-1157-8487, AuthorID 149863; +7 909 146-40-64,s_golovina@yahoo.com

Aleksey V. Ruchkin ${ }^{1}$, candidate of sociological sciences, associate professor, associate professor of the department of management and economic theory, ORCID 0000-0002-6981-3080, AuthorID 615361; +7 909 022-78-24, alexeyruchkin87@gmail.com

Ivan N. Mikolaychik ${ }^{2}$, doctor of agricultural sciences, professor, acting vice-rector for research, ORCID 0000-0001-5189-2174, AuthorID 149861; +7912 522-64-64,min_ksaa@mail.ru

Lidiya N. Smirnova ${ }^{2}$, candidate of pedagogical sciences, leading researcher of the research institute "Study of Agro-Industrial Complex Problems", ORCID 0000-0002-7991-0802, AuthorID 904486; +7 912 212-68-17, lidia-1311@mail.ru

${ }^{1}$ Ural State Agrarian University, Ekaterinburg, Russia

${ }^{2}$ Kurgan State Agricultural Academy, Lesnikovo, Russia

${ }^{3}$ Russian State Agrarian Correspondence University, Moscow, Russia 